\title{
miR-539 suppresses proliferation and induces apoptosis in renal cell carcinoma by targeting high mobility group A2
}

\author{
ZHI-HUA YE and DING-WEN GUI

\begin{abstract}
Department of Urology and Hubei Key Laboratory of Kidney Disease Pathogenesis and Intervention, Huangshi Central Hospital, Affiliated Hospital of Hubei Polytechnic University,
\end{abstract} \\ Edong Healthcare Group, Huangshi, Hubei 435000, P.R. China
}

Received April 2, 2017; Accepted September 6, 2017

DOI: $10.3892 / \mathrm{mmr} .2018 .8578$

\begin{abstract}
Renal cell carcinoma (RCC) is one of the most common urinary malignancies with a high rate of morbidity. MicroRNAs (miRNAs) have been shown to be critical post-transcriptional regulators in tumorigenesis. The present study aimed to investigate the effect of miRNA (miR)-539 on the proliferation and apoptosis of RCC. The expression of miR-539 and high mobility group AT-hook 2(HMGA2) were examined in clinical RCC specimens. The 786-O RCC cell line was also used and was transfected with miR-539 mimics or inhibitors. The correlation between miR-539 and HMGA2 was confirmed using a luciferase reporter assay. Cell viability and apoptosis were detected using MTT and flow cytometry assays. The protein levels of HMGA2, AKT, phosphorylated (p)-AKT, mammalian target of rapamycin (mTOR) and p-mTOR were analyzed using western blot analysis. The results revealed that miR-539 was negatively correlated with the expression of HMGA2 in clinical RCC specimens. Further experiments identified HMGA2 as a direct target of miR-539. The overexpression of miR-539 downregulated the expression of HMGA2, reduced cell proliferation and promoted cell apoptosis, whereas the knockdown of miR-539 led to the opposite results. miR-539 also suppressed the phosphorylation of AKT and mTOR, without altering the levels of total AKT and mTOR. Taken together, the results of the present study indicated that miR-539 negatively regulated the expression of HMGA2 in clinical specimens and in vitro. miR539 inhibited cell proliferation and induced apoptosis in RCC cells. This regulatory effect of miR-539 may be associated with the AKT
\end{abstract}

Correspondence to: Dr Ding-Wen Gui, Department of Urology and Hubei Key Laboratory of Kidney Disease Pathogenesis and Intervention, Huangshi Central Hospital, Affiliated Hospital of Hubei Polytechnic University, Edong Healthcare Group, 16 North Guilin Road, Huangshi, Hubei 435000, P.R. China E-mail: zhy_1982zhy@126.com

Key words: microRNA-539, high mobility group AT-hook 2, renal cell carcinoma, proliferation, apoptosis signaling pathway. Therefore, miR-539 may be used as a biomarker for predicting the progression of RCC.

\section{Introduction}

Renal cell carcinoma (RCC) is a common malignancy and is the third leading cause of cancer-associated mortality in cancer of the urinary system (1). The morbidity rates of RCC continue to increase rapidly, whereas the overall five-year survival rate has not improved significantly, resulting in unsatisfactory prognosis (2). Therefore, examining the molecular interactions occurring in the initiation and progression of RCC may assist in identifying more therapeutic targets and investigating novel approaches in prognosis.

High mobility group AT-hook 2 (HMGA2) is a member of the HMGA group, which functions as a transcriptional enhancer in the alteration of chromatin structure via binding to AT-rich regions in DNA sequences (3). HMGA2 is an oncofetal protein, the expression of which is at low levels or absent with the differentiation of tissues, which is in contrast to its high level of expression with the dedifferentiation of several types of malignant tumor $(4,5)$. Notably, it has been observed that the abnormal expression of HMGA2 shows high correlation with various malignancies, including cancer of the colon, breast, kidney, pancreas, liver and lung $(3,6-8)$. Previous studies have demonstrated that the level of HMGA2 is positively correlated with tumor size and Fuhrman grade in RCC, and the high expression of HMGA2 leads to poor prognosis of patients, which indicates that HMGA2 is important in the progression of RCC (7).

MicroRNAs (miRNAs) are a series of non-coding, single-stranded RNA molecules, consisting of 20-24 nucleotides (9). They are endogenously expressed and negatively correlated with the transcription of genes through binding to their 3'-untranslated-region (3'UTR) (10). Previous studies have demonstrated that the aberrant expression of miRNAs contributes to tumor growth and is involved in regulating the physiological behavior of tumor cells through the modulation of target genes at mRNA or protein levels. For example, miRNA-129 (miR-129) inhibits the metastasis of prostate cancer by binding to centriolar coiled-coil protein 110 (11), miR-200a regulates the proliferation and metastasis of pancreatic cancer through modulating the DEK gene (12), 
and miR-543 is downregulated in colorectal cancer samples, acting as tumor suppressor by targeting KRAS, MTA1 and HMGA2 (13).

In the present study, the discrepant expression between miR-539 and HMGA2 was analyzed in RCC tumor tissues, and it was predicted and confirmed that miR-539 binds to HMGA2. Subsequently, the regulatory effect of miR-539 on the proliferation and apoptosis of RCC cells was confirmed. These findings suggest that miR-539 may be important in the progression of RCC and may be used as a biomarker for predicting the growth of RCC.

\section{Materials and methods}

Clinical specimens. The present study was approved by the Institutional Ethics Committee of Ren'min Hospital Affiliated to Wuhan University (Wuhan, China) and performed according to the guidelines on ethical management. Tissue specimens from a 23 cases of RCC and 19 paired normal tissues were collected from the Department of Urology, Ren'min Hospital of Wuhan University between 2015 and 2017. Written informed consent was signed by all participants prior to the study. The tumor stage and grade were classified according to the tumor-node-metastasis staging system of the American Joint Committee on Cancer (14). All tissues were divided into two sections, one of which was fixed in $4 \%$ paraformaldehyde, and the other was immediately frozen and stored at $-80^{\circ} \mathrm{C}$ for subsequent analysis. The clinical data of the participants are shown in Table I.

Cell lines and cell culture. The 786-O human kidney cancer cell line was purchased from the American Type Culture Collection (Manassas, VA, USA) and cultured in minimal Roswell Parker Memorial Institute-1640 medium (Gibco; Thermo Fisher Scientific, Inc., Waltham, MA, USA) supplemented with $10 \%$ FBS (Wuhan Biofavor Biotech Services Co. Ltd., Wuhan, China) at $37^{\circ} \mathrm{C}$ under normoxic conditions (5\% $\mathrm{CO}_{2}, 95 \% \mathrm{O}_{2}$ ).

Transfection and plasmid construction. The 786-O cells were transfected with miR-539 mimics and miR-539 inhibitors (Biossci Biotechnology Co., Ltd., Wuhan, China) using Lipofectamine 2000 (Invitrogen; Thermo Fisher Scientific, Inc.) according to the manufacturer's protocol. A total of $1.0 \times 10^{6}$ cells $/ \mathrm{ml}$ cells were transferred to 24 -well plates for group comparison experiments. The cells were serum-starved for $24 \mathrm{~h}$ for further analyses prior to reaching a confluence of $90 \%$.

The wild-type sequence of the HMGA2 3'UTR containing predicted miR-539 binding site was amplified from the 786-O cells using the polymerase chain reaction (PCR) method. The mutant 3'UTR sequence of HMGA2 was produced using an overlap-extension PCR method. Subsequently, the wild-type and mutant sequences were subcloned into a psiCHECK-2 vector (Promega Corporation, Madison, WI, USA).

In silico prediction. The binding of miR-539 to HMGA2 was predicted using open access databases TargetScan (www.targetscan.org), miRanda (www.microrna. org). and miRwalk2.0 (http://zmf.umm.uni-heidelberg. de/apps/zmf/mirwalk2/), and a putative binding site in the 3'UTR of HMGA2 for miR-539 was identified (Fig. 2C).

Luciferase reporter assays. For the luciferase reporter assay, the 786-O cells were seeded into a 24 -well plate $\left(2 \times 10^{3}\right.$ cells per well), and then co-transfected with the miR-539 mimics and HMGA2-3'UTR-luciferase plasmids. At 48 h post-transfection, the cells were collected and lysed. The luciferase activities were analyzed using a Dual-Luciferase Reporter Assay system (Promega Corporation).

Immunohistochemistry. The expression of HMGA2 was examined using immunohistochemical staining. The tissues were sliced into $3-\mu \mathrm{m}$ sections and deparaffinized in xylene, followed by dehydration in gradient ethanol and blocking with $3 \%$ hydrogen peroxide for $15 \mathrm{~min}$ at room temperature. The sections were then incubated with rabbit polyclonal anti-HMGA2 (cat. no. ab97276; 1:500; Abcam, Cambridge, UK) antibodies at $4^{\circ} \mathrm{C}$ overnight. Following washing three times with PBS, the sections were stained using diaminobenzidine reagents and counterstained with hematoxylin and the results were observed with an Olympus BX50 light microscope (Olympus Corporation, Tokyo, Japan).

Western blot analysis. Total cellular proteins were extracted with radioimmunoprecipitation buffer (Beyotime Institute of Biotechnology, Jiangsu, China). The protein concentration was measured using a bicinchoninic acid assay (Beyotime Institute of Biotechnology). Briefly, equivalent quantities of protein sample (40 $\mu \mathrm{g} /$ lane) were separated by $10 \%$ sodium dodecyl sulfate-polyacrylamide gel electrophoresis and subsequently electrotransferred onto polyvinylidene fluoride membranes (Bio-Rad Laboratories, Inc., Hercules, CA, USA). Subsequently, the membrane was blocked at $4^{\circ} \mathrm{C}$ for $1 \mathrm{~h}$ with TBS containing $0.05 \%$ Tween-20 (TBST) buffer with $5 \%$ non-fat milk and then incubated with the following primary antibodies against HMGA2 (1:1,000; cat. no. ab97276; Abcam), AKT (1:1,000; cat. no. sc-8312; Santa Cruz Biotechnology, Inc., Dallas, TX, USA), phosphorylated (p)-AKT (1:1,000; cat. no. sc-33437; Santa Cruz Biotechnology, Inc.), mammalian target of rapamycin (1:500, mTOR; cat. no. sc-8319; Santa Cruz Biotechnology, Inc.) and p-mTOR (1:500, cat. no. sc-101738; Santa Cruz Biotechnology, Inc.) at $4{ }^{\circ} \mathrm{C}$ overnight. Following incubation with secondary antibodies conjugated with horseradish peroxidase (LK2001 and LK2003; 1:100; Sungene Biotech, Co., Ltd., Tianjin, China) for $1 \mathrm{~h}$ at room temperature, the bands were examined using an enhanced chemiluminescence system (MultiSciences Biotech Co., Ltd., Hangzhou, China).

Reverse transcription-quantitative PCR (RT-qPCR). Total RNA was extracted from the clinical specimens using TRIzol reagent (Invitrogen; Thermo Fisher Scientific, Inc.) according to the manufacturer's protocol. Subsequently, the RNAs were reversed transcribed into cDNA using a reverse transcription reagent kit (Takara Biotechnology Co., Ltd., Dalian China). Subsequently, cDNA was amplified using an SYBR Green mix kit and the ABI 7900 Real-Time PCR system (Applied Biosystems; Thermo Fisher Scientific, Inc.) following the manufacturer's instructions. PCR was performed with the 
Table I. Clinicopathological features of participants.

\begin{tabular}{|c|c|c|c|c|c|}
\hline \multirow[b]{2}{*}{ Variables } & \multirow[b]{2}{*}{ Group } & \multicolumn{3}{|c|}{ HMGA2 expression (n) } & \multirow[b]{2}{*}{ P-value } \\
\hline & & High & Low & Total & \\
\hline \multirow{2}{*}{ Gender } & Male & 7 & 6 & 13 & \multirow[t]{2}{*}{0.509} \\
\hline & Female & 4 & 6 & 10 & \\
\hline \multirow{2}{*}{ Age (years) } & $<60$ & 8 & 9 & 17 & \multirow[t]{2}{*}{0.901} \\
\hline & $\geq 60$ & 3 & 3 & 6 & \\
\hline \multirow{2}{*}{ Clinical stage } & Stage I-II & 5 & 10 & 15 & \multirow{2}{*}{0.056} \\
\hline & Stage III-IV & 6 & 2 & 8 & \\
\hline \multirow[t]{2}{*}{ T classification } & T1-2 & 4 & 10 & 14 & \multirow[t]{2}{*}{0.021} \\
\hline & T3-4 & 7 & 2 & 9 & \\
\hline \multirow[t]{2}{*}{ Lymph node metastasis } & No & 7 & 11 & 18 & \multirow[t]{2}{*}{0.103} \\
\hline & $\mathrm{N} 1-2$ & 4 & 1 & 5 & \\
\hline \multirow[t]{2}{*}{ M classification } & M0 & 9 & 11 & 20 & \multirow[t]{2}{*}{0.483} \\
\hline & M1-2 & 2 & 1 & 3 & \\
\hline \multirow[t]{2}{*}{ Differentiation degree } & Well-moderate & 4 & 12 & 16 & \multirow[t]{2}{*}{0.007} \\
\hline & Low & 6 & 1 & 7 & \\
\hline
\end{tabular}

T, tumor; M, metastasis.

following thermocycling conditions: Initial denaturation at $94^{\circ} \mathrm{C}$ for $4 \mathrm{~min}, 40$ cycles of $94^{\circ} \mathrm{C}$ for $30 \mathrm{sec}, 56^{\circ} \mathrm{C}$ for $30 \mathrm{sec}$ and $72^{\circ} \mathrm{C}$ for $25 \mathrm{sec}$, using the ABI 7900 Real-Time PCR system (Applied Biosystems; Thermo Fisher Scientific Inc.). The primer sequences are shown in Table II. Relative levels of miR-539 and mRNA expression levels of HMGA2 were normalized to that of small nuclear RNA U6 (for miRNAs) or GAPDH (for mRNAs) respectively. The relative expression of miRNA or mRNA was calculated using the $2^{-\Delta \Delta \mathrm{Ct}}$ method (15).

Cell proliferation assay. The 786-O cells were cultured in 96 -well plates $\left(2 \times 10^{3}\right.$ cells per well) for $24 \mathrm{~h}$. The cells were then stained with $10 \mu \mathrm{l}$ of $5 \mathrm{mg} / \mathrm{ml}$ MTT per well (Sigma-Aldrich; Merck Millipore; Darmstadt, Germany) for $4 \mathrm{~h}$ at $37^{\circ} \mathrm{C}$. The culture medium was then discarded and $150 \mu \mathrm{l}$ of dimethyl sulfoxide was added. The absorbance was detected at $490 \mathrm{~nm}$ with an ELX-800 spectrometer reader (Bio-Tek Instruments, Inc., Winooski, VT, USA).

Cell apoptosis assay. Cell apoptosis was measured using Annexin V-fluorescein isothiocyanate (FITC)/propidium iodide (PI) staining (BD Pharmingen, San Diego, CA, USA) according to the manufacturer's protocol. In brief, the 786-O cells were collected in 6-well plates at a concentration of $10^{5}$ cells/ml. Annexin V-FITC (5 $\left.\mu \mathrm{l}\right)$ and PI $(5 \mu \mathrm{l})$ were then distributed into each well, and the cells were incubated in the dark for 15 min to undergo flow cytometry (BD LSRII; BD Pharmingen).

Statistical analysis. All data are presented as the mean \pm standard deviation. Differences were assessed by two-tailed Student's t-test and $\chi^{2}$ test, as appropriate. $\mathrm{P} \leq 0.05$ was considered to indicate a statistically significant difference.
Table II. Primers for reverse transcription-quantitative polymerase chain reaction analysis.

\begin{tabular}{ll}
\hline Gene & \multicolumn{1}{c}{ Primer sequence (5'-3') } \\
\hline HMGA2 & F: CGAAAGGTGCTGGGCAGCTCCGG \\
& R: CCATTTCCTAGGTCTGCCTCTTG \\
miR-539 & F: GGAGAAAUUUCCUUGUGUGU \\
& R: UUUCUUUAAAGGAACAUACA \\
U6 snRNA & F: CTCGCTTCGGCAGCACATATACT \\
& R: ACGCTTCACGAATTTGCGTGTC \\
GAPDH & F: TGAAGGTCGGTGTGAACGGATTTGGTC \\
& R: CATGTAGGCCATGAGGTCCACCAC \\
\hline
\end{tabular}

HMGA2, HMGA2, high mobility group AT-hook 2; miR, microRNA; snRNA, small nuclear RNA; F, forward; R, reverse.

All experiments were performed at least three times. Statistical analyses were performed using SPSS 19.0 (IBM SPSS, Armonk, NY, USA).

\section{Results}

Expression of miR-539 negatively correlates with the expression of HMGA2 in RCC samples. To examine the expression of HMGA2 in RCC samples and adjacent normal control samples, immunohistochemistry was performed to stain tissues from 23 cases of RCC tissues and 19 paired normal tissues. As shown in Fig. 1A, HMGA2 was predominantly expressed in the nucleus of cells and the expression of HMGA2 was significantly increased in the RCC tissues, compared with that in the adjacent normal tissues. In addition to the progression 
A
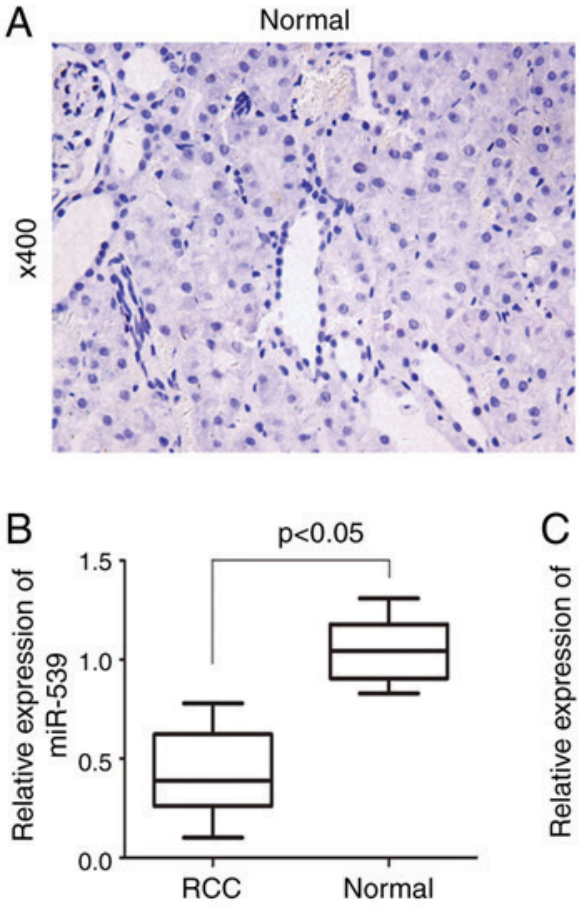

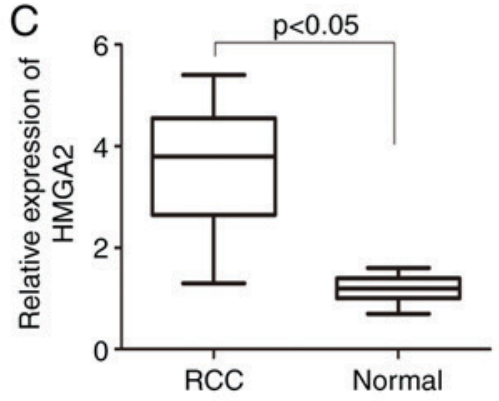

Renal cell carcinoma
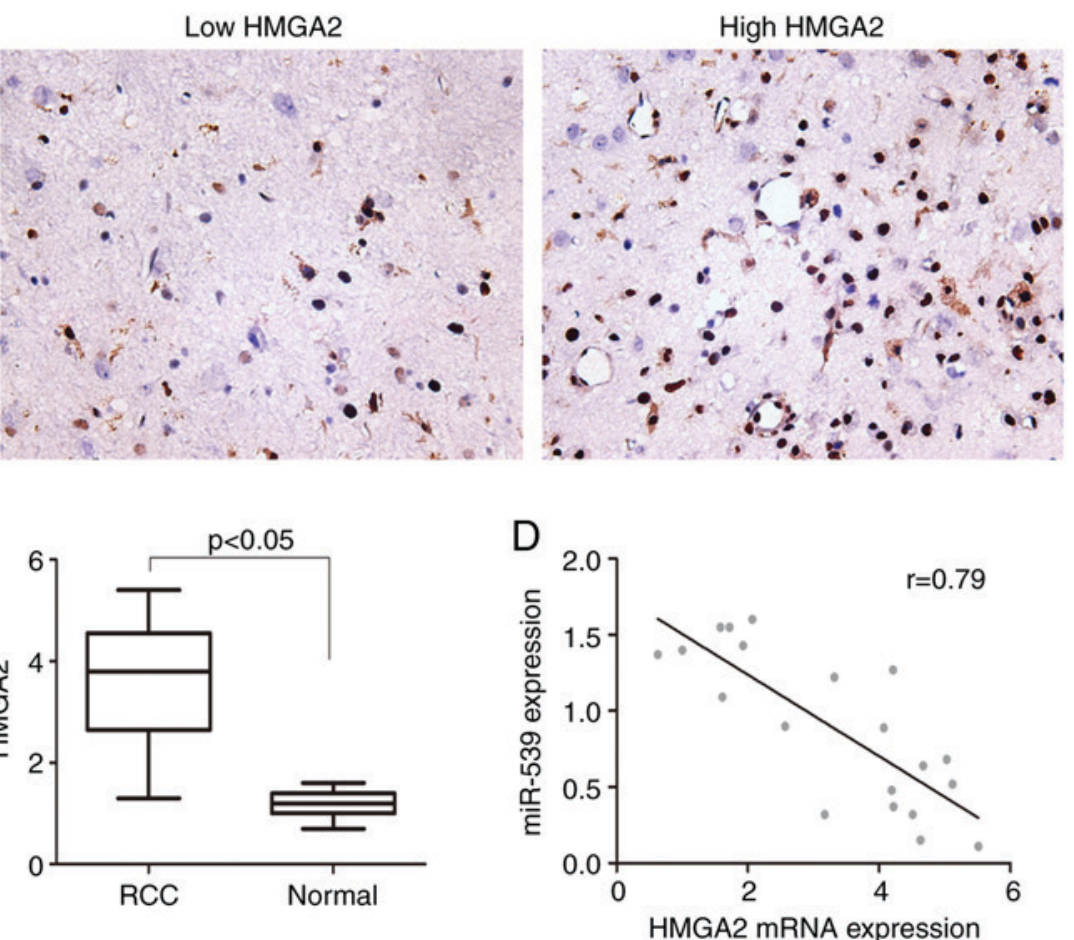

Figure 1. Expression of miR-539 is negatively correlated with the expression of HMGA2 in RCC samples. (A) Immunohistochemical staining of HMGA2 in normal renal tissues, low clinical stage RCC tissues and high clinical stage RCC tissues. Box plots represent relative expression of (B) miR-539 and (C) HMGA2 in clinical samples, detected using reverse transcription-quantitative polymerase chain reaction analysis. (D) Clinical specimens analysis suggested that miR-539 was inversely correlated with the expression of HMGA2. Data are presented as the mean \pm standard deviation of three independent experiments. RCC, renal cell carcinoma; HMGA2, high mobility group AT-hook 2; miR, microRNA.

of RCC, HMGA2 was expressed at a higher level in advanced samples. By contrast, the RT-qPCR results revealed a lower expression of miR-539 in RCC samples, compared with that in paired normal samples (Fig. 1B and C). Pearson's correlation analysis was performed, and the result showed that the expression of miR-539 was negatively correlated with the expression of HMGA2 (Fig. 1D).

miR-539 directly regulates the expression of HMGA2. To further confirm the hypothesis that miR-539 downregulates the expression of HMGA2, the 786-O RCC cell line was used. The 786-O cells were transfected with miR-539 mimics or inhibitors to obtain cells with miR-539 overexpression or knockdown. Using western blot analysis, the expression of HMGA2 was measured. As the data revealed, the expression of HMGA2 was significantly downregulated in the cells overexpressing miR-539, but was moderately increased in the miR-539-knockdown cells (Fig. 2A and B). Subsequently, it was predicted that miR-539 was an upstream regulator of HMGA2 using open access databases and a putative binding site in the 3'UTR of HMGA2 for miR-539 was identified (Fig. 2C). To confirm this prediction, a luciferase reporter assay was performed. The result revealed that the reporter activity of the HMGA2 3'UTR was significantly suppressed in the cells overexpressing miR-539. However, this effect was abrogated when the putative binding site in the 3'UTR of HMGA2 was mutated (Fig. 2D). Taken together, these results suggested that miR-539 negatively regulated the expression of HMGA2 by directly targeting it.
miR-539 suppresses proliferation and promotes apoptosis of $R C C$ cells. To investigate the effect of miR-539 on the proliferation of RCC cells, an MTT assay was performed and the data showed that the viability of 786-O cells transfected with miR-539 mimics was markedly suppressed, compared with that in the control group, whereas transfection with miR-539 inhibitors increased cell viability (Fig. 3A). Subsequently, the effect of miR-539 on the apoptosis of RCC cells was observed. The results of the flow cytometry revealed that the overexpression of miR-539 led to a significant increase in apoptotic rate, compared with that in the control group, and this promotion of cell apoptosis was reversed following the use of miR-539 inhibitor (Fig. 3B and C). Collectively, these results indicated that miR-539 regulated the proliferation and apoptosis of RCC cells.

Effects of miR-539 on the AKT signaling pathway. To investigate whether the AKT signaling pathway was involved in the potential mechanism of miR-539, the present study detected the phosphorylation levels of AKT and mTOR. The results of the western blot analysis showed that the overexpression of miR-539 suppressed the expression of p-AKT and p-mTOR, compared with the expression in the control group, whereas the knockdown of miR-539 led to the opposite results. The total levels of AKT and mTOR were not affected by miR-539 (Fig. 4A and B). These data indicated that miR-539 is involved in regulation of the AKT signaling pathway, which may be an important factor to the growth of 786-O cells. 
A

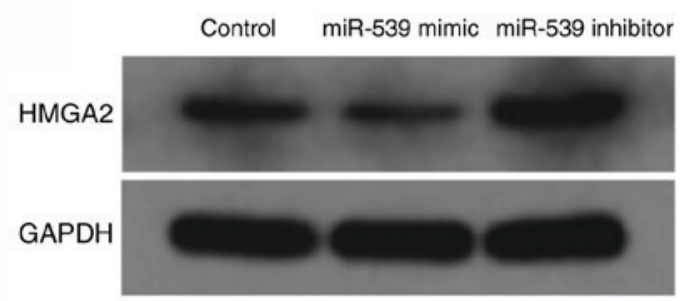

C

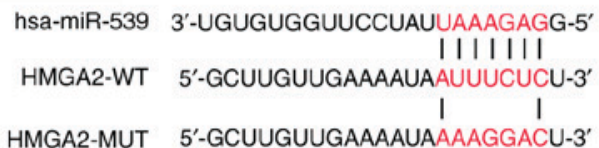

B
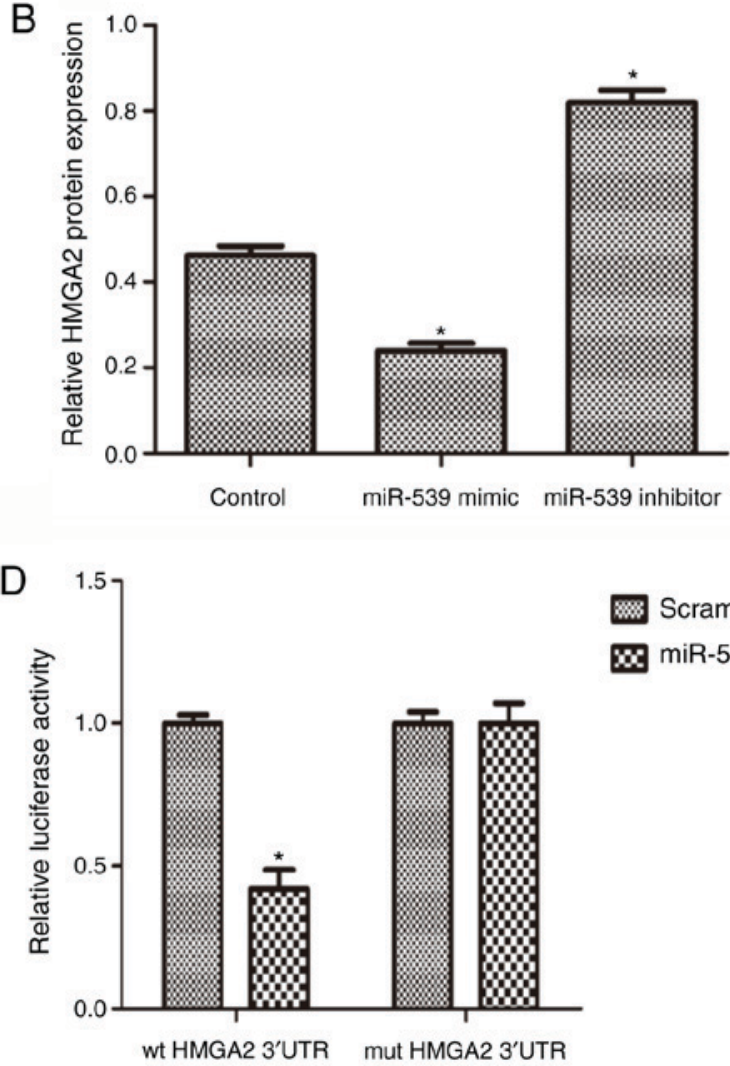

Scramble

$\mathbf{X}$

miR-539

Figure 2. Expression of miR-539 is negatively correlated with the expression of HMGA2 in renal cell carcinoma samples. (A) Western blot analysis and (B) quantification revealed that the protein level of HMGA2 increased significantly in the cells overexpressing miR-539, compared with that in the miR-539-knockdown cells. (C) Sequence alignment of the predicted miR-539 binding sites with the HMGA2 3'UTR and the mutated sequence of miR-539. (D) Luciferase reporter assay was performed in 786-O cells co-transefected with miR-539 mimics and reporter vectors containing the HMGA2 3'UTR or mutated HMGA2 3'UTR. Relative luciferase activities are shown. Data are presented as the means \pm standard deviation of three independent experiments ("P $<0.05$, compared with the control). miR, microRNA; HMGA2, high mobility group AT-hook 2; wt, wild-type; mut, mutant; 3'UTR, 3'untranslated region.
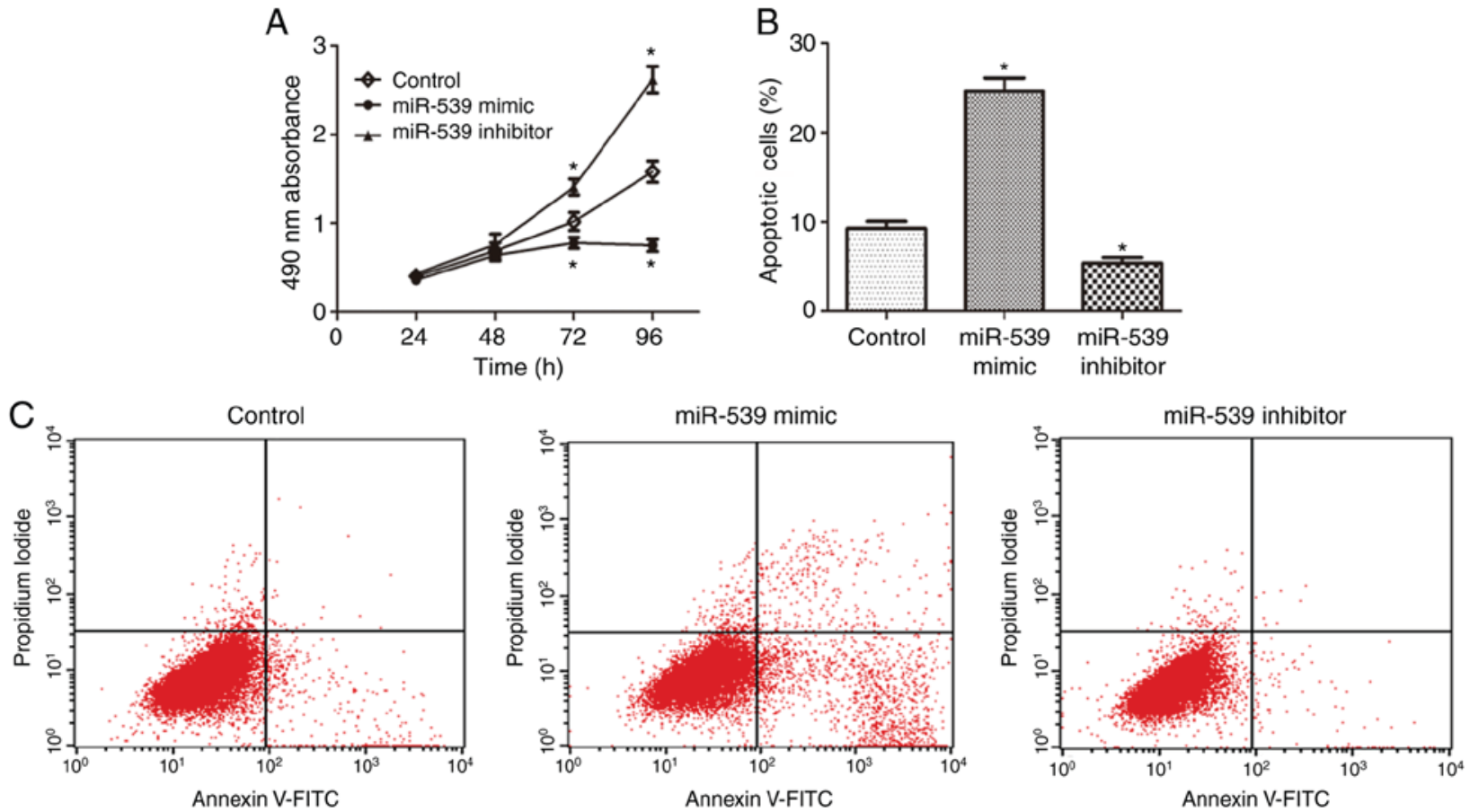

Figure 3. miR-539 suppresses the proliferation and promotes the apoptosis of renal cell carcinoma cells. (A) An MTT assay was performed to analyze cell viability among the miR-539-overexpression, miR-539-knockdown and control groups. The absorbance value was examined at 24, 48, 72 and $96 \mathrm{~h}$ following transfection. Flow cytometry was performed $48 \mathrm{~h}$ post-transfection. (B) Apoptotic cell rates are shown in histograms determined from (C) flow cytometry data. Data are presented as the mean \pm standard deviation of three independent experiments ("P $<0.05$, compared with the control). miR, microRNA; FITC, fluorescein isothiocyanate. 
A

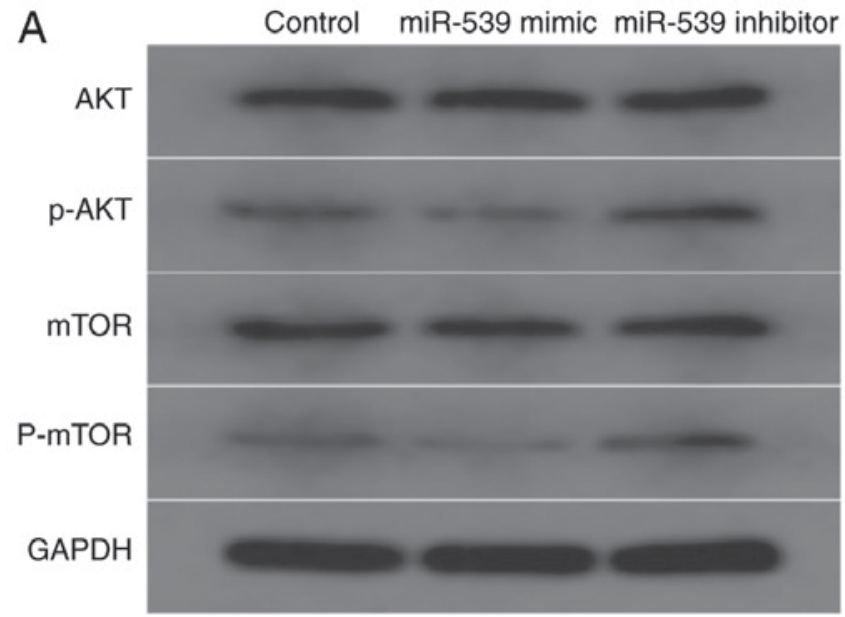

$\mathrm{B}$

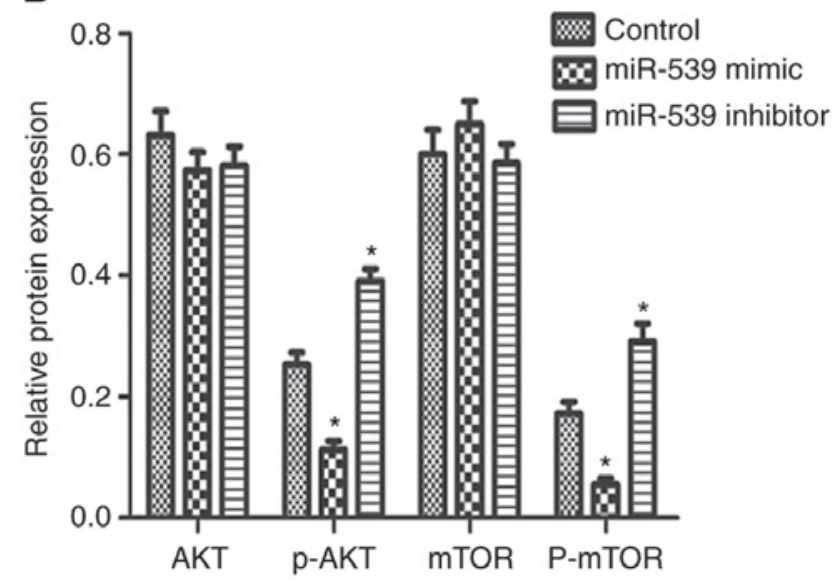

Figure 4. (A) Effects of miR-539 on the AKT signaling pathway. (B) The protein levels of AKT, p-AKT, mTOR and p-mTOR were examined using western blot analysis. GAPDH was used as an internal control. Data are presented as the mean \pm standard deviation of three independent experiments ( $\mathrm{P}<0.05$, compared with the control). miR, microRNA; mTOR, mammalian target of rapamycin; p-, phosphorylated.

\section{Discussion}

RCC is not sensitive to radiotherapy or chemotherapy; therefore, partial or radical nephrectomy is the preferred treatment for localized RCC in patients $(16,17)$. Unfortunately, RCC usually occurs without typical symptoms, therefore, it is difficult to diagnose prior to its development into advanced stages or distant metastasis (18). According to previous studies, up to one-third of patients with RCC exhibit metastatsis at the time of diagnosis, whereas almost $40 \%$ of patients with a localized lesion have been shown to experience recurrence following surgical therapies $(19,20)$. Therefore, it is important to elucidate the mechanism underlying the progression of RCC and examine novel molecular interactive targets to develop more effective therapeutic approaches. Previous studies have indicated that miRNA can regulate tumor proliferation and apoptosis in cancer cells by targeting specific genetic markers (21). Based on these results, the present study aimed to identify miRNAs involved in regulating the progression of RCC.

Several studies have confirmed that miRNA is important in tumorigenesis and metastasis. It has been elucidated that miRNA regulates gene expression post-transcriptionally and acts as an oncogene or tumor suppressor in different types of cancer $(22,23)$. miR-539 has been reported to be downregulated in osteosarcoma and suppresses tumor metastasis by targeting MMP-8 (24). It has also been shown that miR-539 functions as a tumor suppressor in prostate cancer through binding to sperm-associated antigen 5 (25). A previous study revealed that miR-539 can be used as a prognostic biomarker for colon cancer (26). However, the effect of miR-539 on RCC and its underlying mechanisms had not been elucidated. In the present study, a negative correlation was found between miR-539 and HMGA2 in RCC specimens. Compared with adjacent normal tissues, miR-539 was significantly downregulated, whereas HMGA2 was upregulated in tumors. Subsequently, the binding correlation between miR-539 and HMGA2 was predicted using open access databases, and HMGA2 was confirmed as a direct downstream target of miR-539 using a luciferase reporter assay.
HMGA2 has been reported to be abnormally expressed in various types of cancer, and to regulate the proliferation and apoptosis of tumor cells through multiple pathways $(27,28)$. For example, HMGA2 promotes the proliferation and metastasis of nasopharyngeal cancer cells through activation by the transforming growth factor- $\beta /$ small mothers against decapentaplegic 3 signaling pathway (29). In addition, the downregulation of HMGA2 effectively inhibits the proliferation process and promotes apoptosis in prostate cancer (30). In the present study, in vitro experiments were performed using the 786-O renal carcinoma cell line to simulate the progression of RCC. As expected, the overexpression of miR-539 suppressed the expression of HMGA2, whereas the inhibition of miR-539 caused a significant upregulation in the expression of HMGA2. These data demonstrated that miR-539 negatively regulated the expression of HMGA2 in RCC cells, which was in agreement with the observations from clinical samples.

A previous study showed that the phosphoinositide3-kinase/-Akt signaling pathway is involved in the regulation of cell proliferation and apoptosis $(31,32)$. It has also been reported that HMGA2 promotes cell proliferation by activating the AKT pathway (33). Accordingly, the present study aimed to investigate whether the AKT signaling pathway mediated the tumor suppression induced by miR-539. The data revealed that the overexpression of miR-539 inhibited the phosphorylation of AKT and mTOR, rather than altering the expression of total AKT and mTOR. Taken together, the above results suggested that miR-539 may be important in regulating the AKT pathway. This regulatory effect may be initiated via the modulation of the expression of HMGA2 by miR-539.

In conclusion, the present study indicated that miR-539 acted as a tumor suppressor in RCC cells by suppressing cell proliferation and inducing cell apoptosis, and this suppression may be due, at least in part, to the modulation of HMGA2 through the AKT signaling pathway. These results suggested that miR-539 may be used as a diagnostic biomarker for RCC treatment. 


\section{Acknowledgements}

Not applicable.

\section{Funding}

No funding was received.

\section{Availability of data and materials}

The analyzed data sets generated during the study are available from the corresponding author on reasonable request.

\section{Authors' contributions}

ZY was responsible for conception and design of the study, data collection and analysis, and manuscript writing. DG, designed the study, performed critical revision and supervised all phases of the study.

\section{Ethics approval and consent to participate}

The present study was approved by the Institutional Ethics Committee of Ren'min Hospital Affiliated to Wuhan University (Wuhan, China) and performed according to the guidelines on ethical management. Written informed consent was signed by all participants prior to the study.

\section{Consent for publication}

Written informed consent was signed by all participants prior to the study.

\section{Competing interests}

The authors declare that they have no competing interests.

\section{References}

1. Siegel R, Ward E, Brawley O and Jemal A: Cancer statistics, 2011: The impact of eliminating socioeconomic and racial disparities on premature cancer deaths. CA Cancer J Clin 61: 212-236, 2011.

2. Yang CM, Ji S, Li Y, Fu LY, Jiang T and Meng FD: $\beta$-Catenin promotes cell proliferation, migration, and invasion but induces apoptosis in renal cell carcinoma. Onco Targets Ther 10: 711-724, 2017.

3. Wu J, Zhang S, Shan J, Hu Z, Liu X, Chen L, Ren X, Yao L, Sheng H, Li L, et al: Elevated HMGA2 expression is associated with cancer aggressiveness and predicts poor outcome in breast cancer. Cancer Lett 376: 284-292, 2016.

4. Piscuoglio S, Zlobec I, Pallante P, Sepe R, Esposito F, Zimmermann A, Diamantis I, Terracciano L, Fusco A and Karamitopoulou E: HMGA1 and HMGA2 protein expression correlates with advanced tumour grade and lymph node metastasis in pancreatic adenocarcinoma. Histopathology 60: 397-404, 2012.

5. Parameswaran S, Xia X, Hegde G and Ahmad I: Hmga2 regulates self-renewal of retinal progenitors. Development 141: 4087-4097, 2014.

6. Pallante P, Sepe R, Puca F and Fusco A: High mobility group a proteins as tumor markers. Front Med (Lausanne) 2: 15, 2015.

7. Na N, Si T, Huang Z, Miao B, Hong L, Li H, Qiu J and Qiu J: High expression of HMGA2 predicts poor survival in patients with clear cell renal cell carcinoma. Onco Targets Ther 9: 7199-7205, 2016.
8. Huang W, Li J, Guo X, Zhao Y and Yuan X: miR-663a inhibits hepatocellular carcinoma cell proliferation and invasion by targeting HMGA2. Biomed Pharmacother 81: 431-438, 2016.

9. Bushati N and Cohen SM: microRNA functions. Annu Rev Cell Dev Biol 23: 175-205, 2007.

10. Kala R, Peek GW, Hardy TM and Tollefsbol TO: MicroRNAs: An emerging science in cancer epigenetics. J Clin Bioinforma 3: 6, 2013.

11. Bijnsdorp IV, Hodzic J, Lagerweij T, Westerman B, Krijgsman O, Broeke J, Verweij F, Nilsson RJ, Rozendaal L, van Beusechem VW, et al: miR-129-3p controls centrosome number in metastatic prostate cancer cells by repressing CP110. Oncotarget 7: 16676-16687, 2016.

12. Wu X, Wu G, Wu Z, Yao X and Li G: MiR-200a suppresses the proliferation and metastasis in pancreatic ductal adenocarcinoma through downregulation of DEK gene. Transl Oncol 9: 25-31, 2016.

13. Fan C, Lin Y, Mao Y,Huang Z, Liu AY, Ma H, Yu D, Maitikabili A, Xiao H, Zhang C, et al: MicroRNA-543 suppresses colorectal cancer growth and metastasis by targeting KRAS, MTA1 and HMGA2. Oncotarget 7: 21825-21839, 2016.

14. Martínez-Salamanca JI, Huang WC, Millán I, Bertini R, Bianco FJ, Carballido JA, Ciancio G, Hernández C, Herranz F, Haferkamp A, et al: Prognostic impact of the 2009 UICC/AJCC TNM staging system for renal cell carcinoma with venous extension. Eur Urol 59: 120-127, 2011.

15. Rao X, Huang X, Zhou Z and Lin X: An improvement of the $2^{\wedge}$ (-delta delta CT) method for quantitative real-time polymerase chain reaction data analysis. Biostat Bioinforma Biomath 3: 71-85, 2013.

16. Chow TF, Youssef YM, Lianidou E, Romaschin AD, Honey RJ, Stewart R, Pace KT and Yousef GM: Differential expression profiling of microRNAs and their potential involvement in renal cell carcinoma pathogenesis. Clin Biochem 43: 150-158, 2010.

17. Pan XD, Gu DH, Mao JH, Zhu H, Chen X, Zheng B and Shan Y: Concurrent inhibition of mTORC1 and mTORC2 by WYE-687 inhibits renal cell carcinoma cell growth in vitro and in vivo. PLoS One 12: e172555, 2017.

18. Wu SW, Chen PN, Lin CY, Hsieh YS and Chang HR: Everolimus suppresses invasion and migration of renal cell carcinoma by inhibiting FAK activity and reversing epithelial to mesenchymal transition in vitro and in vivo. Environ Toxicol 32: 1888-1898, 2017.

19. Ko JJ, Xie W, Kroeger N, Lee JL, Rini BI, Knox JJ, Bjarnason GA, Srinivas S, Pal SK, Yuasa T, et al: The international metastatic renal cell carcinoma database consortium model as a prognostic tool in patients with metastatic renal cell carcinoma previously treated with first-line targeted therapy: A population-based study. Lancet Oncol 16: 293-300, 2015.

20. Chatzizacharias NA, Rosich-Medina A, Dajani K, Harper S, Huguet E, Liau SS, Praseedom RK and Jah A: Surgical management of hepato-pancreatic metastasis from renal cell carcinoma. World J Gastrointest Oncol 9: 70-77, 2017.

21. Jiang T, Li M, Li Q, Guo Z, Sun X, Zhang X, Liu Y, Yao W and Xiao P: MicroRNA-98-5p inhibits cell proliferation and induces cell apoptosis in hepatocellular carcinoma via targeting IGF2BP1. Oncol Res 25: 1117-1127, 2017.

22. Okato A, Goto Y, Kurozumi A, Kato M, Kojima S, Matsushita R, Yonemori M, Miyamoto K, Ichikawa T and Seki N: Direct regulation of LAMP1 by tumor-suppressive microRNA-320a in prostate cancer. Int J Oncol 49: 111-122, 2016.

23. Lin Y, Liu AY, Fan C, Zheng H, Li Y, Zhang C, Wu S, Yu D, Huang Z, Liu F, et al: MicroRNA-33b Inhibits Breast Cancer Metastasis by Targeting HMGA2, SALL4 and Twistl. Sci Rep 5: 9995, 2015.

24. Jin $\mathrm{H}$ and Wang W: MicroRNA-539 suppresses osteosarcoma cell invasion and migration in vitro and targeting Matrix metallopeptidase-8. Int J Clin Exp Pathol 8: 8075-8082, 2015.

25. Zhang H, Li S, Yang X, Qiao B, Zhang Z and Xu Y: miR-539 inhibits prostate cancer progression by directly targeting SPAG5. J Exp Clin Cancer Res 35: 60, 2016.

26. Bobowicz M, Skrzypski M, Czapiewski P, Marczyk M, Maciejewska A, Jankowski M, Szulgo-Paczkowska A,Zegarski W, Pawłowski R, Polańska J, et al: Prognostic value of 5-microRNA based signature in T2-T3N0 colon cancer. Clin Exp Metastasis 33: 765-773, 2016.

27. Zou Q, Xiong L, Yang Z, Lv F, Yang L and Miao X: Expression levels of HMGA2 and CD9 and its clinicopathological significances in the benign and malignant lesions of the gallbladder. World J Surg Oncol 10: 92, 2012. 
28. Li Y, Zhao Z, Xu C, Zhou Z, Zhu Z and You T: HMGA2 induces transcription factor Slug expression to promote epithelial-to-mesenchymal transition and contributes to colon cancer progression. Cancer Lett 355: 130-140, 2014.

29. Xia YY, Yin L, Jiang N, Guo WJ, Tian H, Jiang XS, Wu J, Chen $\mathrm{M}, \mathrm{Wu} \mathrm{JZ}$ and $\mathrm{He} \mathrm{X}$ : Downregulating HMGA2 attenuates epithelial-mesenchymal transition-induced invasion and migration in nasopharyngeal cancer cells. Biochem Biophys Res Commun 463: 357-363, 2015.

30. Shi Z, Wu D, Tang R, Li X, Chen R, Xue S, Zhang C and Sun X: Silencing of HMGA2 promotes apoptosis and inhibits migration and invasion of prostate cancer cells. J Biosci 41: 229-236, 2016.

31. Xie HX, Xu ZY, Tang JN, DU YA, Huang L, Yu PF and Cheng XD: Effect of Huaier on the proliferation and apoptosis of human gastric cancer cells through modulation of the PI3K/AKT signaling pathway. Exp Ther Med 10: 1212-1218, 2015.
32. Deng W, Zhang Y, Gu L, Cui J, Duan B, Wang Y and Du J: Heat shock protein 27 downstream of P38-PI3K/Akt signaling antagonizes melatonin-induced apoptosis of SGC-7901 gastric cancer cells. Cancer Cell Int 16: 5, 2016.

33. Tan L, Wei X, Zheng L, Zeng J, Liu H, Yang S and Tan H: Amplified HMGA2 promotes cell growth by regulating Akt pathway in AML. J Cancer Res Clin Oncol 142: 389-399, 2016.

(†) $\ominus$ This work is licensed under a Creative Commons cc) Attribution-NonCommercial-NoDerivatives 4.0 International (CC BY-NC-ND 4.0) License. 